Proceedings of the National Conference on Neutron Scattering and the Complementary Methods in the Investigations of the Condensed Phases, Chlewiska 2007

\title{
Influence of the Molecular Shape on Its Thermodynamic Functions
}

\author{
A. LEWANDOWSKa AND J. KonARSKI \\ Faculty of Chemistry, A. Mickiewicz University \\ Grunwaldzka 6, 60-780 Poznań, Poland
}

Dedicated to Professor Jerzy Janik on the occasion of his 80th birthday

Free energy and entropy were calculated for molecules of different shapes in gas phase and under small pressure. The values of the thermodynamic functions were found strongly dependent on the molecular shape. The results obtained were applied to predict the route of internal energy deactivation as well as the preferred form of the molecules examined.

PACS numbers: 05.20.--y, 05.70.--a, 33.10.Cs

\section{Introduction}

Macroscopic systems are very well described by their thermodynamic functions such as free energy $(F)$, entropy $(S)$, heat capacity $\left(C_{v}\right.$ or $\left.C_{p}\right)$ and so forth (see for example [1,2]). Values of these functions depend on internal energy such as electronic, vibrational and rotational, as well as on external characterization such as translational motion or potential of interaction between molecules.

Free energy of the system is described by equation

$$
F=F_{\mathrm{t}}+F_{\mathrm{el}}+F_{\text {vib }}+F_{\text {rot }},
$$

where $F_{\mathrm{t}}$ is the translational free energy, $F_{\mathrm{el}}$ is the electronic free energy, $F_{\mathrm{vib}}$ is the vibrational free energy, $F_{\text {rot }}$ is the rotational free energy.

The free energy is calculated from the general formula (see for example [1-3]):

$$
F=-k T \ln Z \text {, }
$$

where

$$
Z=\sum_{k} \exp \left(-\beta e_{k}\right)
$$

where $\beta=(k T)^{-1}$ and $k$ is the Boltzmann constant, $T$ - temperature in kelvin scale, $e_{k}$ is energy of $k$-th element of a system considered.

The entropy and heat capacity are related to the free energy through the well-known equations, namely 
$S=-\frac{\partial F}{\partial T} \quad$ and $\quad C_{v}=T \frac{\partial S}{\partial T}$

Using Eq. (2) we obtain

- the translational free energy

$F_{\mathrm{t}}=-k T \ln Z_{\mathrm{tr}}, \quad Z_{\mathrm{tr}}=\frac{8 \pi^{3} V(2 \pi m k T)^{3 / 2}}{h^{3}}$,

where $V$ is the volume of the system considered, $h$ - the Planck constant, $m$ reduced mass,

- the electronic free energy

$$
F_{\mathrm{el}}=-k T \ln \sum_{\mathrm{el}} \exp \left(-\beta e_{\mathrm{el}}\right)
$$

where $e_{\mathrm{el}}$ is electronic energy,

- the rotational free energy

$$
F_{\text {rot }}=-k T \ln Z_{\text {rot }}, \quad Z_{\text {rot }}=\sum_{J}(2 J+1) \exp \left(-\beta E_{J}\right),
$$

where $E_{J}$ is rotational energy,

- the vibrational free energy

$$
F_{\mathrm{vib}}=-k T \ln Z_{\mathrm{vib}}, \quad Z_{\mathrm{vib}}=\sum_{v} \exp \left(-\beta E_{v}\right)
$$

and $E_{v}$ is vibrational energy.

Solution of the Schrödinger equation for the rotation, vibration, and electronic states gives a relation between the energy and the structure of a system considered (see for example $[4,5]$ and references cited there).

For example for a spherical molecule

$E_{\text {rot }}=B J(J+1)$,

where $B$ is rotational constant, $B=h^{2} /\left(8 \pi^{2} m r_{0}^{2}\right), r_{0}$ is equilibrium distance between atoms in a molecule, $J$ - rotational quantum number and $m$ - reduced mass, whereas for a symmetrical molecule

$$
E_{\mathrm{rot}}=A J(J+1)+(F-A) K^{2},
$$

$A=h^{2} /\left(8 \pi^{2} m r_{1}^{2}\right), F=h^{2} /\left(8 \pi^{2} m r_{2}^{2}\right)$, where $r_{1}$ and $r_{2}$ are geometrical parameters of a symmetrical molecule along $x$ and $z$-axis, respectively.

Introducing Eqs. (8) and (9) into Eq. (6) we are able to calculate free energy for a spherical or symmetrical rotor. On the basis of these equations we obtain different values of free energy for different rotors. The above analysis has shown that different shapes of molecules lead to different values of their thermodynamic functions.

\section{Calculations}

Let us consider a gas phase under small pressure. Under these conditions external effects such as scattering of molecules do not perturb internal, i.e. electronic, rotational, and vibrational states. 
Using these suppositions two thermodynamic functions have been calculated, namely the vibrational and rotational free energy and the vibrational and rotational entropy. The thermodynamic functions for the electronic state take very small values due to a high value of the electronic energy and were neglected. Equation (1) shows that the shape of the molecule influences only the rotational and vibrational component of the overall free energy, so only the values of these two free energies were calculated.

Three different molecules have been chosen for the calculation, namely $\mathrm{C}^{34} \mathrm{~S}$ as a representative of a spherical rotor, acetonitrile $\left(\mathrm{H}_{3} \mathrm{C}-\mathrm{C}=\mathrm{N}\right)$ representing prolate rotor, and ethylaldehyde $\left(\mathrm{H}_{2} \mathrm{C}=\mathrm{CHCOH}\right)$ which has a semispherical structure.

TABLE I

Calculated values (in $\mathrm{kJ} / \mathrm{mol}$ ) of rotational free energy $F_{\text {rot }}$ and entropy $S_{\text {rot }}$, vibrational free energy $F_{\text {vib }}$ and entropy $S_{\text {vib }}$ as well as internal free energy $F_{\text {rot }}+F_{\text {vib }}$ and entropy $S_{\text {rot }}+S_{\text {vib }}$ for spherical molecule.

\begin{tabular}{c|c|c|c|c|c|c}
\hline \hline$T[\mathrm{~K}]$ & $F_{\text {rot }}$ & $F_{\text {vib }}$ & $F_{\text {rot }}+F_{\text {vib }}$ & $S_{\text {rot }}$ & $S_{\text {vib }}$ & $S_{\text {rot }}+S_{\text {vib }}$ \\
\hline 100 & -3.707 & 7.5790 & 3.872 & 0.04535 & 0 & 0.04535 \\
200 & -7.414 & 7.5795 & 0.165 & 0.04537 & 0 & 0.04537 \\
300 & -13.855 & 7.573 & -6.281 & 0.0545 & 0.00014 & 0.5464 \\
500 & -25.213 & 7.468 & -17.745 & 0.07119 & 0.00104 & 0.7224 \\
1000 & -56.187 & 6.103 & -50.083 & 0.0645 & 0.00440 & 0.0689
\end{tabular}

TABLE II

Calculated values (in $\mathrm{kJ} / \mathrm{mol}$ ) of internal free energy $\left(F_{\text {rot }}+F_{\text {vib }}\right)$ for prolate $F_{\mathrm{p}}$ and oblate $F_{\mathrm{o}}$ form, as well as internal entropy $\left(S_{\text {rot }}+S_{\text {vib }}\right)$ for prolate $S_{\mathrm{p}}$ and oblate $S_{\mathrm{o}}$ form of a molecule.

\begin{tabular}{c|c|c|c|c}
\hline \hline$T[\mathrm{~K}]$ & $F_{\mathrm{p}}$ & $F_{\mathrm{o}}$ & $S_{\mathrm{p}}$ & $S_{\mathrm{o}}$ \\
\hline 100 & -4.813 & -6.056 & 0.0728 & 0.0598 \\
200 & -13.361 & -11.409 & 0.0815 & 0.0695 \\
300 & -22.277 & -18.661 & 0.09278 & 0.0747 \\
500 & -40.313 & -34.330 & 0.09654 & 0.08111 \\
1000 & -89.269 & -77.370 & 0.1017 & 0.0898
\end{tabular}

The results of the calculations are shown in Table I, presenting the values of rotational free energy $\left(F_{\text {rot }}\right)$, vibrational free energy $\left(F_{\text {vib }}\right)$, rotational entropy $\left(S_{\text {rot }}\right)$, vibrational entropy $\left(S_{\mathrm{vib}}\right)$ in $\mathrm{kJ} / \mathrm{mol}$ for the spherical rotor, i.e. the $\mathrm{C}^{34} \mathrm{~S}$ molecule. Tables II and III present the data for the prolate $\left(r_{1}>r_{2}\right)\left(\mathrm{H}_{3} \mathrm{C}-\right.$ $\mathrm{C}=\mathrm{N})$ and semispherical $\left(r_{1} \approx r_{2}\right)\left(\mathrm{H}_{2} \mathrm{C}=\mathrm{CHCOH}\right)$ molecules. As follows from the 
TABLE III

Calculated values (in $\mathrm{kJ} / \mathrm{mol}$ ) of internal free energy and internal entropy for semispherical molecule $\left(r_{1} \approx r_{2}\right)$ in their prolate $F_{1}, S_{1}$ and oblate $F_{2}, S_{2}$ form, respectively.

\begin{tabular}{c|c|c|c|c}
\hline \hline$T[\mathrm{~K}]$ & $F_{1}$ & $F_{2}$ & $S_{1}$ & $S_{2}$ \\
\hline 100 & -8.200 & -8.160 & 0.0945 & 0.0940 \\
200 & -18.128 & -18.053 & 0.1031 & 0.1027 \\
300 & -28.710 & -28.596 & 0.10817 & 0.10779 \\
500 & -51.034 & -50.848 & 0.1145 & 0.11416 \\
1000 & -111.078 & -109.927 & 0.1238 & 0.1219
\end{tabular}

experimental data and theoretical calculations (see for example $[6,7]$ and references cited therein), the vibrational and rotational excitation can change the shape of the molecules. Therefore, the question arises which form is more stable at a chosen temperature. The answer is given by the results collected in the tables. The necessary experimental data such as geometry, rotational constants, vibrational frequencies were taken from Landolt-Bornstein [8] and Huber-Herzberg tables [9].

\section{Conclusions}

The results of the calculations performed have permitted drawing the following conclusions:

a) The shape of the molecule can change the values of the thermodynamic functions.

b) For the spherical molecule, an interesting effect is observed, namely energy dissipation takes place through the rotational states because $F_{\text {rot }}-F_{\text {vib }}<0$. The above condition allows a spontaneous transition from the vibrational to rotational states (see for example [1,2]).

c) Table II shows that for all temperatures the prolate form of $\mathrm{H}_{3} \mathrm{C}-\mathrm{C}=\mathrm{N}$ is more stable (the entropy is higher) but the spontaneous transitions from the prolate to the oblate form is possible only at low temperatures. At high temperatures the prolate form is more stable. Table III presents the results of the calculation for the $\mathrm{H}_{2} \mathrm{C}=\mathrm{CHCOH}$ molecule for which $r_{1} \approx r_{2}$. Such a molecule prefers the prolate form and has generally a semispherical shape, and the spontaneous transition from the prolate to the oblate form is forbidden whereas from oblate to prolate is allowed.

d) The above presented simple calculations give a similar picture of internal stability of the molecule as that obtained by much more advanced calculations based on quantum mechanics [10], which indicates that the quasi-classical model based on thermodynamic functions may be still used for analysis of stability of the molecular shape. 
e) Values of thermodynamic functions are close to experimental ones and, as it was shown previously [11], influence of external interactions on internal states may be treated as a perturbation.

\section{References}

[1] P. Atkins, Physical Chemistry, Oxford University Press, Oxford 1998.

[2] S. Gladstone, Theoretical Chemistry, Van Nostrand Co., Princeton 1961.

[3] K. Huang, Statistical Mechanics, Wiley, New York 1963.

[4] G. Herzberg, Molecular Spectra and Molecular Structure, Vols. I-III, Van Nostrand Co., New York 1950.

[5] H.W. Kroto, Molecular Rotational Spectra, Wiley, London 1979.

[6] J. Makarewich, Mol. Phys. 68, 107 (1989).

[7] J. Stanek, J. Konarski, Int. J. Bif. Chaos 10, 257 (2000).

[8] Landolt-Bornstein, Zalenverte und Funktionen, Neue Serie, Grupe II, Band 6, Molekul Konstanten, Springer Verlag, Berlin 1974.

[9] K.P. Huber, G. Herzberg, Molecular Spectra and Molecular Structure, IV, Constants of Two Atomic Molecules, Van Nostrand, Toronto 1979.

[10] J. Konarski, Israel J. Chem. 43, 287 (2003).

[11] J. Konarski, Phase Transit. 80, 511 (2007). 